\title{
Mitochondrially-mediated toxicity of bile acids
}

\author{
Carlos M. Palmeira*, Anabela P. Rolo \\ Department of Zoology, Center for Neurosciences and Cell Biology of Coimbra, University of Coimbra, 3004-517 Coimbra, Portugal
}

Received 15 April 2004; received in revised form 26 May 2004; accepted 2 June 2004

Available online 28 July 2004

\begin{abstract}
In the healthy hepatocyte, uptake of bile acids across the basolateral membrane and export via the canalicular export pump, are tightly coupled. Impairment of bile formation or excretion results in cholestasis, characterized by accumulation of bile acids in systemic blood and within the hepatocyte. When the concentration of bile acids exceeds the binding capacity of the binding protein located in the cytosol of the hepatocyte, bile acids induce apoptosis and necrosis, by damage to mitochondria. Mitochondria play a central role on the toxicity of bile acids. In this article, we review the published literature regarding bile acid effects on cell function, especially at the mitochondrial level. In patients with cholestatic liver disease, the extent of hepatocyte damage caused by intracellular accumulation of bile acids appears to be delayed by ingesting a hydrophilic bile acid. However, its effects on disease progression are not completely clarified. Therefore, identification of the mechanisms of cell injury will be of clinical utility, helping in the development of new therapeutic strategies. The goal of this review is to include a fresh consideration of all possible targets and integrating pathways that are involved in cholestasis, as well as in the benefits of bile acid therapy.
\end{abstract}

(C) 2004 Elsevier Ireland Ltd. All rights reserved.

Keywords: Bile acids; Cholestasis; Mitochondria

\section{Bile acids in health and disease}

Bile acids are the major organic solutes in bile, and under physiological conditions, they are chiefly confined to the enterohepatic circulation. However, in cholestasis (a pathophysiologic condition of the liver defined as an impairment of bile formation) (Javitt, 1982), the enterohepatic circulation is interrupted and bile acids accumulate within the liver.

\footnotetext{
* Corresponding author. Tel.: +351-239-83-4729; fax: +351-239-82-6798.

E-mail address: palmeira@ci.uc.pt (C.M. Palmeira).
}

\subsection{Clinical significance of bile acid synthesis}

Bile acids, and their salts are natural products, fundamental constituents of bile. The determining physiological action of bile acids is the emulsification of bile lipids, in particular cholesterol. In view of this, bile acid synthesis and subsequent excretion in the feces represent the only significant mechanism for the elimination of excess cholesterol. Their emulsifying action also facilitate the intestinal absorption of fat-soluble vitamins and the digestion of dietary triacylglycerols, rendering fats accessible to pancreatic lipases. Moreover, bile is an important route of elimination of environmental toxins, carcinogens, drugs 
and their metabolites. Besides cholesterol, other endogenous compounds and metabolic products, such as bilirubin and hormones, are also excreted in the bile (Nathanson and Boyer, 1991).

\subsection{Bile acid synthesis}

The synthesis of $\mathrm{C}_{24}$ bile acids (which are present in most vertebrates) from cholesterol, in the liver, requires the coordinated actions of a dozen enzymes located in every major compartment of the hepatocyte (Russell and Setchell, 1992). In this pathway, the addition of hydroxyl groups and the oxidation of the side chain, form a soluble end product from cholesterol. Although the pattern of hydroxylation varies between species, the hydroxylation is always on one side of the molecule, having the final product invariably a hydrophobic face and a hydrophilic face, resulting in an amphipathic molecule (Fig. 1). However, the differ- ent bile acids have variable degrees of hydrophobicity and hydrophilicity, which are determined by their biochemical and physicochemical properties (Sarbur et al., 2001).

Bile acids synthesized within hepatocytes, are termed primary bile acids, like the dihydroxylated chenodeoxycholic acid (CDCA) and the more hydrophilic trihydroxylated cholic acid (CA). Within the intestines, C-7 dehydroxylation of the primary bile acids by bacteria, produces secondary bile acids, identified as deoxycholic acid (DCA; from CA) and lithocholic acid (LCA; from CDCA). The $7 \alpha$-hydroxy epimerization of CDCA, results in ursodeoxycholic acid (UDCA), currently used for cholesterol gallstone dissolution therapy and in the treatment of cholestatic liver diseases.

Within hepatocytes, all bile acids, whether primary or secondary, are conjugated at the terminal $\left(\mathrm{C}_{24}\right)$ carboxyl group with glycine or taurine (Falany et al.,

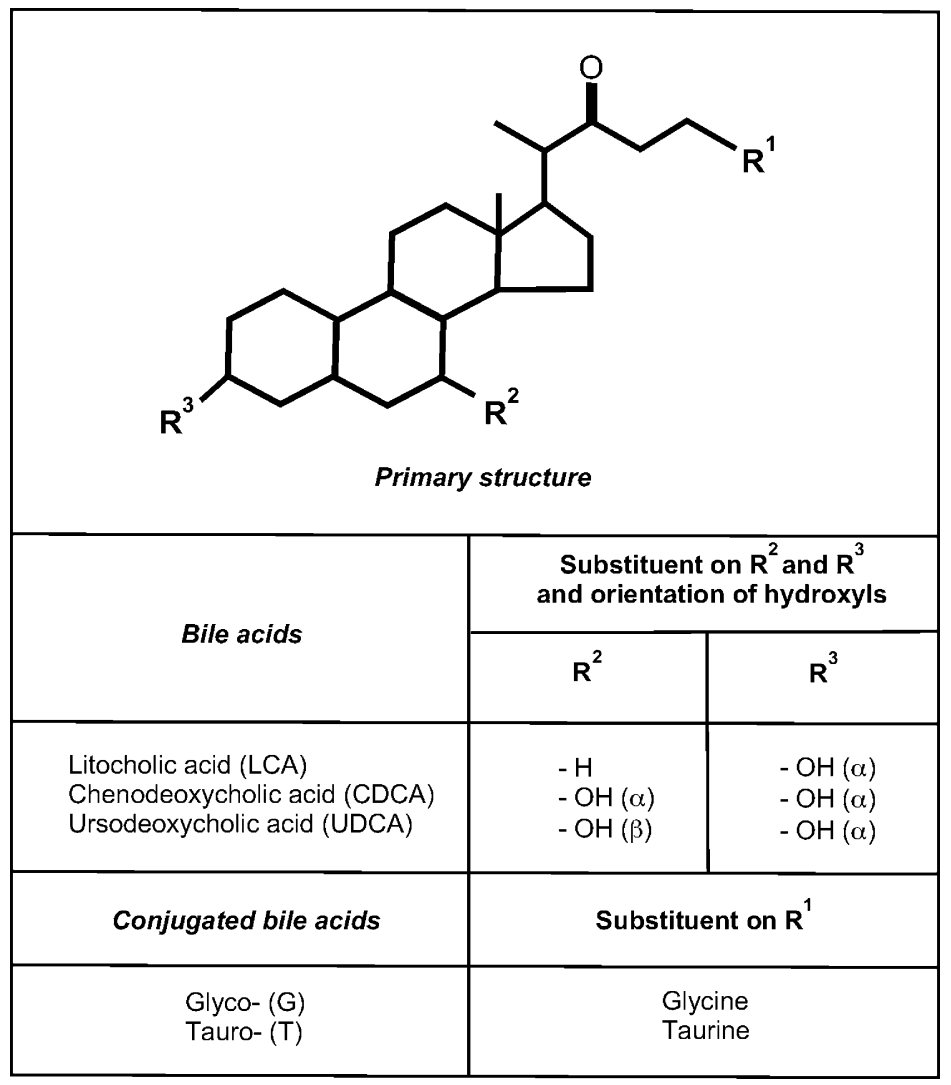

Fig. 1. Structures of major bile acids. 
1994), with the glycine conjugates predominating in man. This amidation increases aqueous solubility at acidic $\mathrm{pH}$, increases resistance to precipitation by calcium and renders bile acids impermeable to cell membranes (Hofmann and Mysels, 1988; Hofmann and Hagey, 1998). Bile acid conjugates exist predominantly in their anionic form at physiological pH (Hofmann, 1994). Therefore, they are called bile salts. For a matter of simplicity we will name both forms as bile acids.

The importance of bile acids as transcriptional regulators of cholesterol homeostasis has becoming increasingly evident. The amount of bile acid synthesized by the liver, is closely regulated to prevent overaccumulation of bile acids on one hand, and cholesterol on the other. When bile acids accumulate beyond physiologic levels, a negative feedback mechanism comes into play, and synthesis is decreased (Russell and Setchell, 1992). Conversely, bile acid synthesis is increased when the substrate, cholesterol, accumulates. Both of these regulatory responses, are transcriptional in nature and are mediated by members of the nuclear hormone receptor family, acting on two genes that encode hydroxylase enzymes in the biosynthetic pathways (Chawla et al., 2001).

\subsection{Bile acid transport}

Bile acids undergo an extensive process of enterohepatic circulation (Müller and Jansen, 1997; Meier and Steger, 2002). The polarized expression of distinct transport systems at the basolateral (sinusoidal) and apical (canalicular) surface domains of hepatocytes, allows an efficient vectorial hepatocellular secretion of bile acids from blood into bile. Basolateral carrier proteins, the $\mathrm{Na}^{+}$-taurocholate cotransport proteins and the $\mathrm{Na}^{+}$-independent organic anion-transporting polypeptides, mediate hepatocellular uptake of bile acids (Fig. 2). Intracellular cytosolic bile acid-binding proteins and, although to a lesser extent, vesicle-associated transport processes, are responsible for the rapid diffusion across hepatocytes for canalicular secretion. Bile acids, are rapidly pumped into the biliary canaliculus by the bile salt export pump, which belongs to the ATP-binding cassette transport superfamily. As bile flows through the bile ducts, it is modified by addition of a watery, bicarbonate-rich secretion from ductal epithelial cells.
Non-ionic diffusion and the apical sodium-dependent bile salt transporter, reabsorb a minor portion of protonated bile acids and monomeric bile salts in bile ductules. The multidrug-resistance-associated proteins, then mediate basolateral export of bile acids from the cholangiocytes (bile duct epithelial cells). Bile acids are carried through bile ducts to the gallbladder, where they are stored for future use. Transcriptional and post-transcriptional regulation of the transporters involved in the enterohepatic circulation, is closely related to the regulation of lipid homeostasis (Fig. 2). Additionally, decreased or even absent expression of hepatocellular transport systems, is an important cause for various cholestatic liver diseases (Trauner et al., 1998).

\subsection{Potential toxicity of bile acids and cholestatic liver diseases}

The impairment of bile formation may be due to drugs or infectious, autoimmune, metabolic, or genetic disorders. Impairment of bile secretion, may result either from a functional defect in bile formation at the level of the hepatocyte (intrahepatic cholestasis), or from an impairment in bile secretion and flow at the level of the biliary tree (extrahepatic cholestasis). Even when the primary insult is to the bile ducts, hepatocellular injury is an invariable feature of cholestasis, associated with the accumulation of bile acids in the liver tissue and blood (Greim et al., 1973; Fischer et al., 1996). The elevated hydrophobic bile acid concentrations will promote liver injury, that ultimately lead to cirrhosis and liver failure, requiring transplantation at a substantial society cost. In severe stages of cholestatic diseases, liver transplantation is the only solution. The recovery of bile excretion is a key early event that determines the survival after transplantation. Impairment of bile flow upon transplantation will promote further liver injury.

The pivotal role of bile acid-mediated hepatotoxicity in the pathogenesis of the disease has been intensively investigated in the last years, being supported by a variety of data from clinical and experimental observations. Although secondary bile acids are extremely toxic, their serum concentrations actually decrease in chronic cholestasis, in contrast to the concentrations of the primary ones, which increase. In healthy individuals, the portal vein serum total bile acid concentration 
(A)

(B)

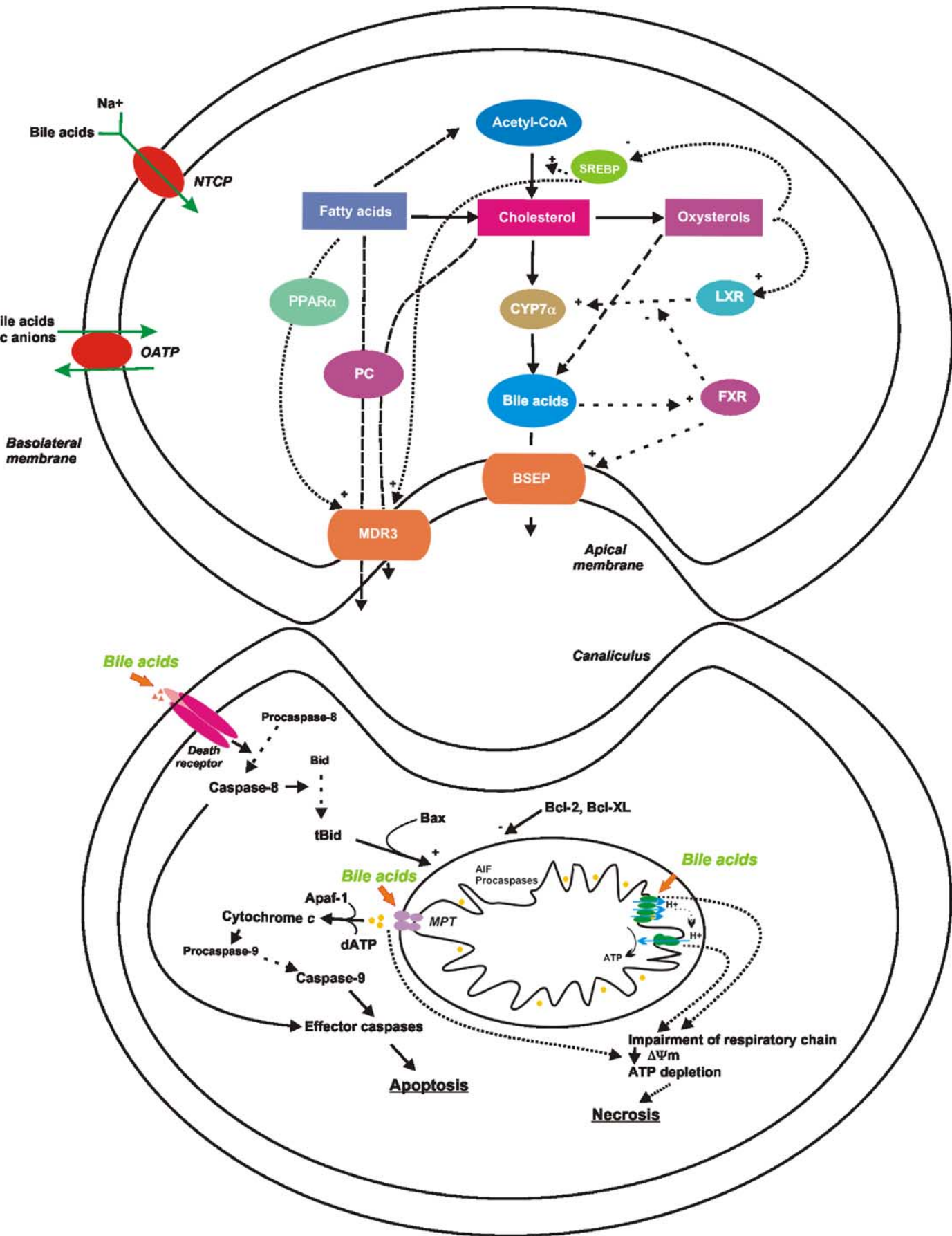

Fig. 2. (A) Hepatocyte bile acid transporters and pathways involved in bile acid metabolism. (B) Cellular dysfunction induced by bile acids: the prominent role of mitochondria. BSEP: bile salt export pump; CYP7 $\alpha$ : cholesterol 7alpha-hydroxylase; FXR: farnesoid X receptor; MDR3: multidrug resistance protein; LXR: liver X receptor; MTP: mitochondrial permeability transition; NTCP: Na ${ }^{+}$-taurocholate transporter; OATP: organic anion-transporting protein; PC: phospholipids; PPAR $\alpha$ : peroxisome proliferator-activated receptor alpha; SREBP: sterol regulatory element binding proteins. 
is approximately $20 \mu \mathrm{M}$; in cholestasis, concentrations can reach $300 \mu \mathrm{M}$ (Ostrow, 1993). Specifically, the concentration of CDCA is significantly increased (Fischer et al., 1996). Hydrophobic bile acids may solubilize membrane-bound lipids, leading to damage to cell membranes. However, at physiologically relevant concentrations, a simple detergent-like action disruption of membrane is probably not relevant (Billington et al., 1980; Fischer et al., 1996).

Taking into consideration the multifaceted role of mitochondria in cell homeostasis, it is natural to hypothesize their role as a primary intracellular target, important in the initiation of cell dysfunction during cholestasis. Although numerous studies have been made in the last years, in order to clarify mechanisms of bile acid hepatotoxicity, one question remains unclear: is mitochondrial damage central and initiator of the dysfunction or it has a secondary role in the cholestatic process, being more a consequence than a trigger? The following overview provides an important insight into the role of mitochondria as a primary intracellular target in the initiation of cell dysfunction during cholestasis.

\section{Mechanisms of bile acid cytotoxicity-mitochondria as a target for bile acid toxicity}

\subsection{Alteration of mitochondrial function by bile acids in isolated mitochondria}

Primary mitochondrial toxicity induced by bile acids has been investigated in order to address its clinical relevance for patients with cholestasis. Studies with isolated rat liver mitochondria have been conducted, providing a reliable basis to predict biochemical and cellular mechanisms that mediate toxicity of bile acids.

Increasing concentrations of the bile acids LCA, DCA, UDCA, CDCA, glycochenodeoxycholic (GCDC) or taurochenodeoxycholic (TCDC), were shown to decrease mitochondrial membrane potential $(\Delta \Psi)$ developed upon succinate energization (Rolo et al., 2000). These compounds also decreased state 3 respiration and enhanced state 4 (Rolo et al., 2000). The decrease in state 3 respiration is probably related with an inhibitory action of these compounds on the phosphorylation system, albeit by a mechanism that has yet to be defined. These observations are in agreement with previous reports (Spivey et al., 1993; Krähenbühl et al., 1994a) which indicates that GCDC, DCA, LCA inhibited state 3 mitochondrial respiration in a dose dependent manner. The described concentration-dependent stimulation of state 4 by LCA, DCA, CDCA, TCDC and GCDC, is associated with an enhanced permeability of mitochondria to protons (Rolo et al., 2000). The observed uncoupling may be a consequence of several interactions of these compounds with mitochondrial structure. TCDC, GCDC, DCA, LCA, and CDCA, induced permeability of the membrane to protons, either by a protonophoric action or by disruption of membrane order.

The majority of compounds possessing protonophoric activity are lipophilic weak acids that have dissociable protons and permeable bilayers either as protonated acids, with $\mathrm{p} K_{\mathrm{a}}$ values in the range of $5-7$, or as the conjugate base. By cycling across the membrane they can catalyze the net electrical uniport of protons and increase the proton conductance of the membrane. In so doing the proton circuit is short-circuited, allowing the process of protonmotive force generation to be uncoupled from ATP synthesis. Although unconjugated bile acids are weak acids, they do not present the structural requirements for uncoupling activity as classical uncouplers such as FCCP, including the presence of an acid-dissociable group, bulky lipophilic groups, and a strong electron-withdrawing moiety. The phenomenology of bile acid-induced uncoupling, could resemble that of fatty acids (which is mediated by integral proteins of the inner mitochondrial membrane), since they are sufficiently hydrophobic to penetrate the lipid membrane. Further studies have shown that CDCA renders mitochondrial membranes more permeable for concentrations as low as $50 \mu \mathrm{M}$, as shown by the increased membrane fluidity probed by DPH (Rolo et al., 2003a). This appears as the specific mechanism by which bile acids stimulate state 4 respiration.

It is well established that hydrophobicity, as demonstrated by its close correlation with the partition coefficient of compounds, is often associated with biological action (Hansch and Dunn, 1972). Bile acids, in particular the hydrophobic dihydroxy, such as CDCA, and monohydroxy species, are surface-active substances and can damage biological membranes (Scholmerich 
et al., 1984). Some reports, regarding in vitro studies, have been shown direct effects of bile acids on membrane fluidity (Isaacson and Van Thiel, 1988; Schroder et al., 1996), associated with increased proton permeability of the membranes (Zhao and Hirst, 1990; Schroder et al., 1996). On the reported studies (Zhao and Hirst, 1990; Schroder et al., 1996), an evaluation of dose-dependence showed that increased membrane fluidity and proton permeability were observed at concentrations as low as $100 \mu \mathrm{M}$. Moreover, it was described that the alterations on membrane fluidity affected specifically the inner membrane.

Induction of the mitochondrial permeability transition (MPT) is known to play a key role in cell death. In isolated liver mitochondria preloaded with calcium, LCA, DCA, CDCA, TCDC, GCDC and UDCA, elicited a concentration-dependent induction of MPT (Rolo et al., 2000), as already described for GCDC and DCA (Botla et al., 1995; Gores et al., 1998). Pretreatment with cyclosporin A (CyA), a specific and potent inhibitor of the MPT (Broekemeier et al., 1989), completely protected against the calcium-dependent swelling. Therefore, MPT induction is involved in bile acid cytotoxicity.

The plasma accumulation of bile acids has been proposed as a causative factor for hepatic cardiomyopathy (Joubert, 1978). Gazawi et al. suggested that bile acid-induced modification of cardiac $\beta$-adrenoreceptor activity might represent a mechanism whereby bile acids exert these effects on cardiac performance (Gazawi et al., 2000). Carvedilol (\{1-[carbazolyl-(4)-oxy]-3-[2-methoxyphenoxyethyl) amino]-propanol-(2)\}) is a lipophilic, vasodilating $\beta$-adrenoreceptor blocking agent, with known cardioprotective properties. Among the various metabolites of carvedilol, BM-910228 (\{1-[3-hydroxycarbazolyl(4)-oxy]-3-[2-methoxyphenoxyethyl)amino]-propanol(2) $\}$ ) is one of the most important. Although there are still considerable uncertainties about the mechanisms underlying the multiple cardioprotective effects of carvedilol, its action could be related with protection of mitochondrial function. Indeed, it has recently been shown that carvedilol inhibits MPT induction by an antioxidant mechanism (Oliveira et al., 2004). Studies have been performed to address if carvedilol potentially prevents mitochondrial dysfunction induced by bile acids. In the presence of calcium and phosphate, CDCA induced the permeability transition in freshly isolated rat liver mitochondria, characterized by membrane depolarization, osmotic swelling, release of matrix calcium and cytochrome $c$, that was prevented by carvedilol (Rolo et al., 2001a,b, 2003b). All these events were blocked by CyA, the calcium uniporter ruthenium red (RR) and carvedilol. Under the same conditions, the hydroxylated metabolite of carvedilol, BM-910228 did not provide any protective effect. Since BM-910228, shown to have higher antioxidant activity than carvedilol (Yue et al., 1994), did not afford protection these results are consistent with a protection by carvedilol that occurs via the inhibition of the MPT, not related with the antioxidant properties (Rolo et al., 2001b).

Additionally, MPT induction by CDCA has been shown to be associated with changes in mitochondrial membrane fluidity (Rolo et al., 2003a), inhibited by carvedilol but not by its hydroxylated analog, BM-910228. So, prevention by carvedilol could be related with the physical stabilization of mitochondrial membrane, as opposed to disturbance induced by CDCA. This would explain the similar results obtained with $\mathrm{CyA}$ and carvedilol. Indeed, membrane fluidity studies, reflecting the dynamic properties of mitochondrial membranes, have shown the strict correlation between the inhibitory effect of carvedilol and the changes in the polarization values due to pore opening (Rolo et al., 2003a). A direct interaction with the pore assembly could also be a hypothesis to explain the action of carvedilol in modulating the ability of CDCA to induce MPT. This difference between carvedilol and metabolite may reflect the different properties of both carvedilol and BM-910228 in terms of membrane insertion. Due to its great lipophilicity, carvedilol has a great tendency for membrane insertion (Cheng et al., 1996) and may disturb the correct assemblage of the proposed pore proteic components.

Interestingly, Rodrigues et al. (Rodrigues et al., 1999, 2000) have shown that hydrophilic bile acids, such as UDCA and its taurine derivative (TUDC), are inhibitors of apoptosis via stabilization of the mitochondrial membrane, through pathways that are independent of the MPT. In fact, these studies regarding induction of apoptosis through a mitochondrial-dependent pathway have showed that although there was no protection by CyA, UDCA or TUDC were able to prevent cell death. The proposed mechanism for protection by hydrophilic bile acids 
correlates inhibition of mitochondrial depolarization and outer membrane disruption, together with modulation of Bax translocation from cytosol to mitochondria. This similarity of cytoprotective actions of both carvedilol and hydrophilic bile acids may reflect modulation of membrane perturbation due to their lipophilicity. In contrast, and in concordance with other reports (Rolo et al., 2000, 2003a), CyA blocked MPT and also prevented mitochondrial swelling and reduced the efflux of cytochrome $c$ induced by DCA, a hydrophobic bile acid (Rodrigues et al., 1999). This suggests that, in contrast to hydrophilic bile acids, cytotoxicity of hydrophobic bile acids involve mitochondrial disruption and cytochrome $c$ release primarily through MPT induction. A study with isolated rat liver mitochondria by Solá et al. (Solá et al., 2002) also described a CyA-sensitive release of cytochrome $c$ induced by hydrophobic bile acids at concentrations lower than the ones necessary to induce structural changes. This supports the hypothesis that mitochondrial toxicity induced by hydrophobic bile acids does not result from generalized damage to the organelle but is the result of specific events.

The central role of mitochondria in the progression of cholestatic injury, upon the initial phase of accumulation of bile acids, was also supported by the differential results upon chemically-induced acute or chronic cholestasis. $\alpha$-naphthylisothiocyanate (ANIT) has been employed as a mean to investigate the developmental aspects of the cholestatic type of hepatotoxic response (Goldfarb et al., 1962; Plaa and Priestley, 1976; Lock et al., 1982). ANIT induces cholestasis in a reproducible and dose-dependent manner following acute administration, bile duct hyperplasia and biliary cirrhosis following chronic administration (Plaa and Priestley, 1976). The cholestasis induced by ANIT is attributed to an increased permeability of the tight junctions between hepatocytes and bile duct epithelial cells, and the subsequent diffusion of osmotically active solutes from the bile into the plasma. Liver mitochondrial bioenergetics was evaluated in $\alpha$-naphthylisothiocyanate (ANIT)-treated Wistar rats (Rolo et al., 2002a), an experimental model of cholestasis. In rats injected with ANIT, liver injury with cholestasis developed within $48 \mathrm{~h}$, as indicated by both serum enzyme activities and total bilirubin concentration. Liver mitochondria isolated from ANIT-treated rats had a higher state 3 respira- tion, respiratory control ratio, ADP/O, and endogenous ATP/ADP ratio, when compared to controls. No change in state 4 respiration was observed. Associated with these parameters, cholestatic mitochondria exhibited an increased resistance to disruption of mitochondrial calcium homeostasis, due to MPT induction. A more efficient mitochondrial function in acute cholestasis, suggested an adaptive response by the hepatocyte to limit liver damage, explaining the slow progression of human cholestatic liver disease. These observations are corroborated by a study reported by Lieser et al., in which evaluation of a specific parameter in mitochondria from bile duct ligated rat, revealed less susceptibility to bile acid-induced MPT (Lieser et al., 1998). Besides modulation of mitochondrial function, liver adapts to cholestasis by attempting to reduce intracellular bile acid concentrations, via regulation of bile acid transporters expression. Decreased expression of the basolateral uptake transporter and maintenance or increased expression of efflux transporters has been documented (Gartung et al., 1996; Lee et al., 2000). Another facet is enhancement of renal excretion of bile acids upon sulfation.

However, after chronic cholestasis, these adaptations do not prevent a persistent, probably irreversible toxic action of bile acids, and disruption of mitochondrial calcium homeostasis is observed (Rolo et al., 2002b). Interestingly, ability of bile acids to induce calcium release from intracellular stores, does not involve solely the mitochondria, as observed in our work, but also the endoplasmic reticulum (Combettes et al., 1988; Rolo et al., 2002b). The rise in intracellular calcium may lead to an increase in calciumdependent proteolysis and, ultimately, cell death.

\subsection{Alterations in cell function upon exposure to bile acids}

Two distinct mechanisms of cell death are triggered by hydrophobic bile acids: necrosis at higher concentrations $(\geq 250 \mu \mathrm{M})$ and apoptosis at lower concentrations $(\leq 100 \mu \mathrm{M})$. To further elucidate mitochondrial role in necrotic cell death induced by toxic bile acids, mitochondrial function, namely the role of the MPT, was evaluated in primary cultures of hepatocytes upon exposure to CDCA, a major contributor to cholestatic liver diseases (Rolo et al., 2003b). Induction of the MPT, inhibition of ATP synthesis and 
consequent metabolic starvation of the cell was the sequence of events triggered by CDCA at $250 \mu \mathrm{M}$, after short time incubation (4 h) (Rolo et al., 2003b). Accordingly, Spivey et al. have previously shown that glycochenodeoxycholate, the glycine conjugate of CDCA, induces lethal hepatocellular injury dependent on ATP depletion (Spivey et al., 1993). Fructose, which is an alternate glycolytic source of cellular ATP, protects against ATP depletion and cell killing induced by CDCA (Rolo et al., 2003b). Because mitochondria are the primary source of ATP in liver cells, these studies indicate that impairment of mitochondrial oxidative phosphorylation is an early and critical event in the mechanism of bile acid cytotoxicity.

The increase in reactive oxygen species (ROS) by bile acids has been largely documented (Sokol et al., 1991, 1995, 1998; Patel and Gores, 1997; Rodrigues et al., 1998; Shivaram et al., 1998). MPT induction and subsequent release of cytochrome $c$ may explain the observations that bile acid cytotoxicity is associated with generation of ROS. Indeed, loss of cytochrome $c$ from the mitochondria, results in impaired electron flow on the respiratory chain, promoting electron leak at the level of ubiquinone-complex III and increased ROS generation. Additionally, the observed decrease in state 3 respiration may be the result of a direct effect on enzyme complexes or due to cytochrome $c$ release induced by bile acids. In this case, oxidative stress does not result from a primary effect of bile acids, but is a secondary phenomenon. Although not involved in the pathogenesis of cholestatic liver injury, high plasma concentrations of lipid peroxides are observed in humans with cholestasis (Lemonnier et al., 1987). A possible adaptation of liver cells to prevent further damage is the up-regulation of glutathione synthesis, as observed in bile duct-ligated rats (Baron and Muriel, 1999). Although administration of antioxidants in this experimental model of cholestasis preserves the normal glutathione/glutathione disulfide (GSH/GSSG) ratio and prevents lipid peroxidation, liver injury, as assessed by serum enzyme activities and tissue histology, was not affected by the treatment (Ballatori and Truong, 1990). This further supports that oxidative stress is a consequence and not a cause of bile acid toxicity.

Hepatocyte apoptosis is a common pathologic feature of cholestatic liver diseases. Interestingly and opposingly to necrosis (Rolo et al., 2002c), at the level of apoptosis induction, cytotoxicity of bile acids does not always correlate with their hydrophobicity. Indeed, apoptosis induction is dependent on the bile acid, its concentration, or its conjugation state. For example, the glycine conjugated of CDCA (GCDC) was shown to induce hepatocyte apoptosis, whereas the taurine conjugate (TCDC) did not (Patel et al., 1994). Rust et al. described that TCDC, but not GCDC, a hydrophobic bile acid, at $50 \mu \mathrm{M}$ activated a phosphatidylinositol 3-kinase-dependent survival pathway in McNtcp.24 rat hepatoma cell line (Rust et al., 2000). A downstream event in this signaling pathway is phosphorylation of Bad, a pro-apoptotic member of the Bcl-2 family (Datta et al., 2002). Moreover, inhibition of this pathway, converted TCDC to a potent apoptotic inducer (Rust et al., 2000). Early studies by Jones et al., pointed that protein kinase $\mathrm{C}$-dependent signaling pathway, plays a critical role in GCDC-induced hepatocyte apoptosis, triggering activation of the protease cathepsin B (Jones et al., 1997).

Thus, depending on the conjugation status of the bile acid, different signaling pathways are recruited to preserve cell viability. Interestingly, treatment of hepatocytes with $50 \mu \mathrm{M}$ DCA, CDCA, and UDCA caused a similar activation of the mitogen-activated protein kinase (MAPK) pathway (Qiao et al., 2002). Partial activation of this pathway provided a significant cytoprotective downstream signal (Qiao et al., 2002), modulating bile acid toxicity.

Studies have been performed to evaluate if mitochondrial dysfunction is a primary pathway for CDCA-induced apoptosis. Upon $24 \mathrm{~h}$, HepG2 cells exposed to $50 \mu \mathrm{M}$ CDCA underwent a significant inhibition of cell proliferation, characterized by an $\mathrm{S}$ phase block (Rolo et al., 2004). Inhibition of cell proliferation by conjugated and unconjugated bile acids, was also observed in rat hepatocytes in primary culture (Martinez-Diez et al., 2000). At $48 \mathrm{~h}$, apoptosis induction by CDCA was observed (Rolo et al., 2004). The observed MPT induction, consequent cytochrome $c$ release and caspase-9 activity, in CDCA-induced apoptosis, are hallmarks of the mitochondrial pathway in apoptotic cell death (Rolo et al., 2004). Another evidence for the role of this pathway in bile acid-induced apoptosis was recently pointed by Graf et al. (Graf et al., 2002). In this study, GCDC induced-cell death in hepatocytes was prevented by betaine, through modulation of cytochrome $c$ release and caspase- 9 activity. 
Inhibition of caspase-9 strongly inhibited caspase-8 activity (Graf et al., 2002), supporting that caspase- 8 activity is a consequence of the early activation of the apoptotic programme at the mitochondrial level.

MPT induction in apoptosis induced by other bile acids has been demonstrated. Yerushalmi et al. have demonstrated that antioxidants prevented GCDCinduced apoptosis in rat hepatocytes, through a mechanism involving the MPT (Yerushalmi et al., 2001). Interestingly, Gumpricht et al. described that the glutathione status of the hepatocyte, altered the cell's response to GCDC-induced necrosis but not apoptosis (Gumpricht et al., 2000). Rodrigues et al. have documented that apoptosis triggered by DCA, involved decreased mitochondrial transmembrane potential and alterations in Bax subcellular distribution, with increased mitochondrial-associated Bax protein levels (Rodrigues et al., 1999). It looks like that MPT induction by bile acids is the initial event, and the initial cytochrome $c$ release stimulates Bax translocation to the mitochondria, accomplishing further cytochrome $c$ release (Rodrigues et al., 1999). In vivo studies show that after bile duct-ligation, Bax, which is normally distributed thoughout the cytoplasmic, mitochondrial, and perinuclear space, translocates to the mitochondria (Oh et al., 2003). The same study described an increase in Bax expression, 3 days after bile duct-ligation and a decrease over time there after. Cytochrome $c$ release was also evident (Oh et al., 2003).

Toxic bile acid-induced hepatocyte apoptosis involves both extrinsic (death receptor-mediated apoptosis) and intrinsic (direct targeting to mitochondria) apoptotic pathways. The intrinsic apoptotic pathway involves direct effects of bile acids on mitochondria with subsequent release of apoptogenic factors as reviewed in this paper. Several studies have demonstrated the importance of death receptor-mediated apoptosis in bile acid cytotoxicity (Miyoshi et al., 1999; Sodeman et al., 2000; Higuchi et al., 2001, 2003). In this pathway, binding to death receptors (Fas pathway) activates procaspase- 8 , which cleaves and activates procaspase- 3 , initiating the caspase cascade (Fig. 2). However, the direct effect of caspase- 8 on effector caspases is relatively small and is amplified by a mitochondrial pathway, in which cytochrome $c$ release is induced by translocation to mitochondria of the cleaved or truncated form of Bid (Fig. 2). Both apoptotic pathways converge at the level of the execution phase, with activation of executioner caspases and cleavage of target substrates.

\section{UDCA therapy: evidence for hepatoprotective effects involving other complementary mechanisms rather than modulation of mitochondrial function}

In recent years, the therapeutic benefit of UDCA has been extensively investigated. Although several studies found improvement in serum markers of liver injury in some patients (van Hoogstraten et al., 1999), other studies found no benefit, especially success rates of liver transplantation and death (Neuberger, 2000). In patients with significantly higher cholestasis indices, the bile acid therapy is more incomplete. Therefore, multiple therapies have been tried (Angulo and Lindor, 1998).

Modulation of mitochondrial membrane perturbation by UDCA and inhibition of MPT induction by other bile acids (Gores et al., 1998; Rodrigues et al., 1998, 1999) have been the mechanisms proposed for UDCA's hepatoprotective properties. So, UDCA has been described as the perfect therapy for cholestatic liver diseases and an anti-apoptotic agent in both hepatocytes and extrahepatic cells (Rodrigues et al., 1998). However other studies have shown potentiation by UDCA of mitochondrial and cell dysfunction induced by other bile acids (Kessel et al., 2000; Rolo et al., 2000, 2003b, 2004; Oyama et al., 2002).

Indeed, UDCA has been shown as a potent inducer of the calcium-dependent MPT, potentiated bile acid-induced necrosis and converted CDCA-induced apoptosis in major necrosis (Rolo et al., 2000, 2002c, 2003b, 2004). Both events were mediated by potentiation of MPT induction, as indicated by the increased mitochondrial depolarization prevented by CyA. Interestingly, UDCA did not increase membrane permeability to protons, supporting that at concentrations as high as $500 \mu \mathrm{M}$ UDCA has no deleterious effect on membranes. No direct effect of UDCA on membrane fluidity or protein order was described by Solá et al. (Solá et al., 2002). This may be the result of the orientation of the steroid hydroxyl groups. Comparing with other bile acids, UDCA, which is the $\beta$-epimer of CDCA, is more hydrophilic. We may recall that, CDCA at concentrations as low as $50 \mu \mathrm{M}$, 
induced increased membrane fluidity (Rolo et al., 2003a). Although being an inducer of the MPT in isolated mitochondria, UDCA $(50 \mu \mathrm{M})$ did not elicit cytochrome $c$ release in HepG2 cells in culture (Rolo et al., 2004). However, isolated hepatocytes exposed to $250 \mu \mathrm{M}$ UDCA underwent a progressive release of lactate dehydrogenase (Rolo et al., 2002c). This effect in cell viability was consequence of UDCA-induced mitochondrial dysfunction through MPT induction. Indeed, an early study by Krähenbühl et al., demonstrated that although UDCA did not impair mitochondrial function up to concentrations of $100 \mu \mathrm{M}$, at $300 \mu \mathrm{M}$, state 3 respiration and respiratory control ratio decreased (Krähenbühl et al., 1994b). Since at this concentration we observed calcium-dependent-MPT induction by UDCA, this could be the mechanism by which UDCA impairs mitochondrial respiration. At this concentration, UDCA also potentiated impairment of mitochondrial respiration induced by CDCA and LCA (Krähenbühl et al., 1994b), as well as MPT induction.

In consequence of UDCA effect on mitochondrial function, it potentiated mitochondrial dysfunction and cell death induced by hydrophobic bile acids. In contrast, previous studies by Rodrigues et al., besides preventing mitochondrial depolarization induced by DCA, UDCA at $500 \mu \mathrm{M}$ also prevents DCA-induced Bax translocation to mitochondria, cytochrome $c$ release and apoptosis induction (Rodrigues et al., 1998, 1999). Additionally, it has also been shown that in vivo DCA feeding is associated with a 4.5 -fold increase in mitochondrial-associated Bax protein levels, while combination feeding with UDCA significantly inhibited these changes (Rodrigues et al., 1998).

A probable explanation for these discrepant results regarding MPT induction by UDCA resides in the preloading of mitochondria with calcium. Exposure of calcium-loaded mitochondria (calcium at a concentration which per se does not induce the MPT) to UDCA results in increased mitochondrial swelling, prevented by CyA (Rolo et al., 2000). In contrast, Rodrigues et al. (Rodrigues et al., 1998, 1999), and also Gores et al. (Gores et al., 1998), evaluate UDCA effects on MPT in a medium in which calcium is absent. So, UDCA alone appears not to cause mitochondrial membrane damage but it lowers the threshold at which calcium induces the MPT. This role of calcium in modulating UDCA effects on the cell is fur- ther supported by UDCA-induced stimulation of calcium release from intracellular stores and subsequent apoptosis activation (Strupp et al., 2000). The activation of a pro-apoptotic cascade by UDCA, which is then blocked by the simultaneous activation of a survival pathway, has also been observed by Qiao et al. (Qiao et al., 2001). However, the role of calcium is not clear, because the apoptotic response of cells exposed to UDCA, was not blocked in a calcium free culture media neither in the presence of an intracellular calcium chelator (Qiao et al., 2001). A possible involvement of sphingomyelinase activation by UDCA (Duan and Nilsson, 1997; Cheng et al., 1999), and subsequent generation of ceramide, may be involved in UDCA-induced cell death (Qiao et al., 2001). Ceramide plays a role in the regulation of cell life and death (Cremesti et al., 2001) and increases mitochondrial membrane permeability.

Other authors have described potentiation of cell dysfunction induced by UDCA. UDCA induces apoptosis in hepatocellular carcinoma cells, mediated by increased levels of Bax in mitochondria and cytochrome $c$ in the cytosol (Oyama et al., 2002). Preincubation of murine leukemia and hepatoma cells with UDCA, potentiated the effects of photodynamic therapy (Kessel et al., 2000). Such action, involved effects at the level of mitochondrial depolarization, release of cytochrome $c$ into the cytosol, and activation of caspase-3. Glyco- and tauro-conjugates of UDCA displayed the same action (Kessel et al., 2000). In contrast, TUDC proved to be effective in reducing both GCDC-induced apoptosis and cytolysis (Benz et al., 1998). The condition that UDCA exerts such an effect, if present before but not upon irradiation (Kessel et al., 2000), supports the suggestion that UDCA per se does not cause mitochondrial membrane damage but lowers the threshold for the action of other compounds.

A new perspective on the hepatoprotective effect of UDCA that has been pointed out, is related with oxidative injury and antioxidant systems in cultured rat hepatocytes. Mitsuyoshi et al., observed that pretreatment with UDCA, significantly prevented the dose-dependent decrease of the viability of the hepatocytes after hydrogen peroxide or cadmium administration (Mitsuyoshi et al., 1999). Such event was correlated with increased amounts of glutathione (GSH) and protein thiols. Additionally, it has also 
been reported, significantly higher mRNA levels of $\gamma$-glutamylcysteine synthetase (a rate-limiting enzyme in glutathione synthesis) and metallothionein in UDCA-treated hepatocytes (Mitsuyoshi et al., 1999). Activation of metallothionein promoter by UDCA was also shown in HepG2 cells (Bernstein et al., 2002) Tanaka et al. reported that, in rats with DCA-induced liver injury, UDCA prevents the decrease in hepatic cytochrome P450 enzymes (Tanaka et al., 1999). It is then suggested that UDCA, is useful for the treatment of liver injury in terms of helping the normalization of the hepatic drug-metabolizing system, which has been shown to be influenced by cholestasis (George et al., 1995; Chen and Farrel, 1996).

Our observations suggest that any therapeutic effect of UDCA must be secondary to an altered membrane transport or non-parenchymal cell function. It has been postulated, that one of the main therapeutic mechanisms of UDCA in cholestatic liver diseases, relates to displacement of the endogenous hepatotoxic bile by expansion of the hydrophilic bile acid pool (i.e., enrichment by UDCA). Exogenously administered UDCA is absorbed and conjugated to taurine and glycine in the liver. The conjugates of UDCA compete with the toxic hydrophobic bile salts for uptake in the enterohepatic circulation. So, UDCA's beneficial effects are generally attributed to its ability to enrich the hepatic bile acid pool, replacing or displacing more hydrophobic bile acids from the hepatocyte. This protective function of UDCA may correlate with competitive displacement of endogenous (i.e., toxic) bile acids either at the level of ileal absorption or at the hepatocyte level (i.e., cell plasma membrane, intracellular organelles). Moreover, UDCA-stimulated calcium entry across the hepatocellular plasma membrane or liberation from cellular calcium stores, is associated with increased vesicular exocytosis of toxic bile salts (Beuers et al., 1993).

UDCA also improves biliary secretion of bile acids, may improve bile flow, and it has immunomodulatory properties that may reduce immune-mediated liver damage. It results in choleresis, with increased capacity of bile duct cells to secrete hydrophobic bile acids, and a reduction in serum levels of CDCA in patients with primary biliary cirrhosis (Beuers et al., 1998). Biliary secretion of phospholipids, which may also be hepatoprotective, appears to be increased with UDCA therapy.

\section{Conclusions}

The data reviewed here demonstrate the key role of mitochondria in bile acid cytotoxicity (Fig. 2).

In isolated mitochondria, hydrophobic bile acids induce alterations in membrane fluidity associated with impairment of mitochondrial respiration and mitochondrial depolarization. MPT induction and subsequent cytochrome $c$ release are also primary events in bile acid toxicity. Such events, unless prevented by efficient mitochondrial protective agents, will progress to cell death. Upon exposure to low bile acid concentrations, release of apoptogenic factors, activation of caspases and DNA fragmentation entails apoptotic cell death. Additionally and besides direct targeting to mitochondria, apoptotic cell death is also induced by bile acids through receptor-dependent-mechanisms.

In acute cholestasis, which may correspond to an initial phase of relatively low concentrations of bile acids accumulated in the hepatocyte, an enhancement of mitochondrial function is probably an adaptive mechanism to limit cell injury. However, high concentrations of bile acids cause a bioenergetic catastrophe culminating in the disruption of plasma membrane integrity. Concomitantly, in chronic cholestasis mitochondrial calcium homeostasis is severely impaired.

Although currently used as a therapeutic agent in cholestatic liver diseases, UDCA displays a toxic character. MPT induction and consequent mitochondrial depolarization are hallmarks of UDCA mitotoxicity. Such effects potentiate cell dysfunction induced by hydrophobic bile acids. Since bile acids used in therapy, do not display the ability to antagonize reactions induced by toxic bile acids as the mechanism by which they might afford cytoprotection against cholestasis, other diverse, complementary array of mechanisms are involved. Improvement of biliary secretion of bile acids and bile flow, expansion of the hydrophilic bile acid pool or immunomodulatory action are hypotheses. Furthermore, after chronic administration of ANIT, our results indicate that UDCA has no role in preventing mitochondrial dysfunction induced by toxic bile acids. A study by Hillstrom et al., also demonstrates that UDCA treatment before and during administration of cholestatic drugs, did not ameliorate cholestatic indices in animal models of cholestasis (Hillstrom et al., 1992). 


\section{Acknowledgements}

We gratefully acknowledge Prof. Kendall Wallace (Department of Biochemistry and Molecular Biology, University of Minnesota-Duluth), for fruitful discussions and the opportunity of developing part of this work at his laboratory. His valuable comments and input, made this work more clear and focus in. This work was partially financed by a Portuguese Research Council-FCT grant (Ref.: POCTI/CBO/42486/2001).

\section{References}

Angulo, P., Lindor, K.D., 1998. Management of primary biliary cirrhosis and autoimmune cholangitis. Clin. Liver Dis. 2, 333351.

Ballatori, N., Truong, A.T., 1990. Cholestasis, altered junctional permeability, and inverse changes in sinusoidal and biliary glutathione release by vasopressin and epinephrine. Mol. Pharmacol. 38, 64-71.

Baron, V., Muriel, P., 1999. Role of glutathione, lipid peroxidation and antioxidants on acute bile-duct obstruction in the rat. Biochim. Biophys. Acta 1472, 173-180.

Benz, C., Angermuller, S., Tox, U., Kloters-Plachky, P., Riedel, H.D., Sauer, P., Stremmel, W., Stiehl, A., 1998. Effect of tauroursodeoxycholic acid on bile-acid-induced apoptosis and cytolysis in rat hepatocytes. J. Hepatol. 28, 99106.

Bernstein, C., Payne, C.M., Bernstein, H., Garewal, H., 2002. Activation of the metallothionein IIA promoter and other key stress response elements by ursodeoxycholate in HepG2 cells: relevance to the cytoprotective function of ursodeoxycholate. Pharmacology 65, 2-9.

Beuers, U., Nathanson, M.H., Isales, C.M., Boyer, J.L., 1993. Tauroursodeoxycholic acid stimulates hepatocellular exocytosis and mobilizes extracellular $\mathrm{Ca}^{2+}$ mechanisms defective in cholestasis. J. Clin. Invest. 92, 2984-2993.

Beuers, U., Boyer, J.L., Paumgartner, G., 1998. Ursodeoxycholic acid in cholestasis: potential mechanisms of avtion and therapeutic applications. Hepatology 28, 1449-1453.

Billington, D., Evans, C.E., Godfrey, P.P., Coleman, R., 1980. Effects of bile salts on the plasma membranes of isolated rat hepatocytes. Biochem. J. 188, 321-327.

Botla, R., Spivey, J.R., Aguilar, H., Bronk, S.F., Gores, G.J., 1995. Ursodeoxycholate (UDCA) inhibits the mitochondrial membrane permeability transition induced by glycochenodeoxycholate: a mechanism of UDCA cytoprotection. J. Pharmacol. Exp. Ther. 272, 930-938.

Broekemeier, K.M., Dempsey, M.E., Pfeiffer, D.R., 1989. Cyclosporin A is a potent inhibitor of the inner membrane permeability transition in liver mitochondria. J. Biol. Chem. 264, 7826-7830.
Chawla, A., Repa, J.J., Evans, R.M., Mangelsdorf, D.J., 2001. Nuclear receptors and lipid physiology: opening the X-files. Science 294, 1866-1870.

Chen, J., Farrel, G.C., 1996. Bile acids produce a generalized reduction of the catalytic activity of cytochromes P450 and other hepatic microsomal enzymes in vitro: relevance to drug metabolism in experimental cholestasis. J. Gastroenterol. Hepatol. 11, 870-877.

Cheng, H.-Y., Randall, C.S., Holl, W.W., Constantinides, P.P., Yue, T.-L., Feuerstein, G.Z., 1996. Carvedilol-liposome interaction: evidence for strong association with the hydrophobic region of the lipid bilayers. Biochim. Biophys. Acta 1284, 20-28.

Cheng, Y., Tauschel, H.D., Nilsson, A., Duan, R.D., 1999. Ursodeoxycholic acid increases the activities of alkaline sphingomyelinase and caspase-3 in the rat colon. Scand. J. Gastroenterol. 34, 915-920.

Combettes, L., Dumont, M., Berthon, B., Erlinger, S., Claret, M., 1988. Release of calcium from the endoplasmic reticulum by bile acids in rat liver cells. J. Biol. Chem. 263, 2299-2303.

Cremesti, A., Paris, F., Grassme, H., Holler, N., Tschopp, J., Fuks, Z., Gulbins, E., 2001. Ceramide enables Fas to cap and kill. J. Biol. Chem. 276, 23954-23961.

Datta, S.R., Ranger, A.M., Lin, M.Z., Sturgill, J.F., Ma, Y.C., Cowan, C.W., Dikkes, P., Korsmeyer, S.J., Greenberg, M.E., 2002. Survival factor-mediated BAD phosphorylation raises the mitochondrial threshold for apoptosis. Dev. Cell 3, 631-643.

Duan, R.D., Nilsson, A., 1997. Purification of a newly identified alkaline sphingomyelinase in human bile and effects of bile salts and phosphatidylcholine on enzyme activity. Hepatology 26, 823-830.

Falany, C.N., Johnson, M.R., Barnes, S., Diasio, R.B., 1994. Glycine and taurine conjugation of bile acids by a single enzyme. Molecular cloning and expression of human liver bile acid CoA: amino acid $N$-acyltransferase. J. Biol. Chem. 269, 19375-19379.

Fischer, S., Beuers, U., Spengler, U., Zwiebel, F.M., Koebe, H.-G., 1996. Hepatic levels of bile acids in end-stage chronic cholestatic liver disease. Clin. Chim. Acta 2511, 173-186.

Gartung, C., Ananthanarayanan, M., Rahman, M.A., Schuele, S., Nundy, S., Soroka, C.J., Stolz, A., Suchy, F.J., Boyer, J.L., 1996. Down-regulation of expression and function of the rat liver $\mathrm{Na}^{+} /$bile acid cotransporter in extrahepatic cholestasis. Gastroenterology 110, 199-209.

Gazawi, H., Ljubuncic, P., Cogan, U., Hochgraff, E., Ben-Shachar, D., Bomzon, A., 2000. The effects of bile acids on $\beta$-adrenoreceptors, fluidity, and the extent of lipid peroxidation in rat cardiac membranes. Biochem. Pharmacol. 59, 16231628.

George, J., Murray, M., Byth, K., Farrel, G.C., 1995. Differential alterations of cytochrome $\mathrm{P}-450$ proteins in livers from patients with severe chronic liver disease. Hepatology 21, 120-128.

Goldfarb, S., Singer, E.J., popper, H., 1962. Experimental cholangitis due to alpha-naphthyl-isothiocyanate (ANIT). Am. J. Pathol. 40, 685-698.

Gores, G.J., Miyoshi, H., Botla, R., Aguilar, H.I., Bronk, S.F., 1998. Induction of the mitochondrial permeability transition as a mechanism of liver injury during cholestasis: a role for 
mitochondrial proteases. Biochim. Biophys. Acta 1366, 167175.

Graf, D., Kurz, A.K., Reinehr, R., Fischer, R., Kircheis, G., Haussinger, D., 2002. Prevention of bile acid-induced apoptosis by betaine in rat liver. Hepatology 36, 829-839.

Greim, H., Czygan, P., Schaffner, F., Popper, H., 1973. Determination of bile acids in needle biopsies of human liver. Biochem. Med. 8, 280-286.

Gumpricht, E., Devereaux, M.W., Dahl, R.H., Sokol, R.J., 2000. Glutathione status of isolated rat hepatocytes affects bile acid-induced cellular necrosis but not apoptosis. Toxicol. Appl. Pharmacol. 164, 102-111.

Hansch, C., Dunn III, W.J., 1972. Linear relationships between lipophilic character and biological activity of drugs. J. Pharmacol. Sci. 61, 1-19.

Higuchi, H., Bronk, S.F., Takikawa, Y., Werneburg, N., Takimoto, R., El-Deiry, W., Gores, G.J., 2001. The bile acid glycochenodeoxycholate induces trail-receptor 2/DR5 expression and apoptosis. J. Biol. Chem. 276, 38610-386188.

Higuchi, H., Yoon, J.H., Grambihler, A., Werneburg, N., Bronk, S.F., Gores, G.J., 2003. Bile acids stimulate cFLIP phosphorylation enhancing TRAIL-mediated apoptosis. J. Biol. Chem. 278, 454-461.

Hillstrom, J., Duane, W.C., Eckfeldt, J.H., Furne, J., Levitt, M.D., 1992. Lack of benefit of ursodeoxycholic acid in drug-induced cholestasis in the rat. Exp. Biol. Med. 200, 122-126.

Hofmann, A.F., Mysels, K.J., 1988. Bile salts as biological surfactants. Colloids Surf. 30, 145-173.

Hofmann, A., 1994. Bile acids. In: Arias, I.M., Boyer, J.L., Fausto, N., Jakoby, W.B., Schacter, D.A., Shafritz, D.A. (Eds.), The Liver: Biology and Pathobiology. Raven Press, New York, pp. 678-710.

Hofmann, A.F., Hagey, L.R., 1998. Bile acids and biliary disease: peaceful coexistence versus deadly warfare. In: Blum, H.E., Bode, C., Sartor, R.B. (Eds.), Gut and Liver. Kluwer Academic Publishers, Lancaster, pp. 85-103.

Isaacson, Y., Van Thiel, D.H., 1988. Increased membrane fluidity and cholestasis: associated but not linked consequences of estrogen treatment. J. Lab. Clin. Med. 112, 663666.

Javitt, N.B., 1982. Bile acids and hepatobiliary disease. In: Schiff, L., Schiff, E.R. (Eds.), Diseases of the Liver. Lippincott, Philadelphia, pp. 119-150.

Jones, B.A., Rao, Yi-P., Stravitz, T., Gores, G.J., 1997. Bile salt-induced apoptosis of hepatocytes involves activation of protein kinase C. Am. J. Physiol. 272, G1109-G1115.

Joubert, P., 1978. Cholic acid and the heart: in vitro studies of the effect on heart rate and myocardial contractility in the rat. Clin. Exp. Pharmacol. Physiol. 5, 9-16.

Kessel, D., Caruso, J.A., Reiners Jr., J.J., 2000. Potentiation of photodynamic therapy by ursodeoxycholic acid. Cancer Res. 60, 6985-6988.

Krähenbühl, S., Talos, C., Sven, F., Reichen, J., 1994a. Toxicity of bile acids on the electron transport chain in isolated rat liver mitochondria. Hepatology 19, 471-479.

Krähenbühl, S., Fischer, S., Talos, C., Reichen, J., 1994b. Ursodeoxycholate protects oxidative mitochondrial metabolism from bile acid toxicity: dose-response study in isolated rat liver mitochondria. Hepatology 20, 1595-1601.

Lee, J.M., Trauner, M., Soroka, C.J., Stieger, B., Meier, P.J., Boyer, J.L., 2000. Expression of the bile salt export pump is maintained after chronic cholestasis in the rat. Gastroenterology 118, 163-172.

Lemonnier, F., Cresteil, D., Feneant, M., Couturier, M., Bernard, O., Alagille, D., 1987. Plasma lipid peroxides in cholestatic children. Acta Paediatr. Scand. 76, 928-934.

Lieser, M.J., Park, J., Natori, S., Jones, B.A., Bronk, S.F., Gores, G.J., 1998. Cholestasis confers resistance to the rat liver mitochondrial permeability transition. Gastroenterology 115, 693-701.

Lock, S., Lavigne, J., Plaa, G.L., 1982. The effect of alphanaphthylisothiocyanate on bile secretion prior to and during the onset of cholestasis in the rat. Toxicol. Lett. 10, 50-57.

Martinez-Diez, M.C., Serrano, M.A., Monte, M.J., Marin, J.J., 2000. Comparison of the effects of bile acids on cell viability and DNA synthesis by rat hepatocytes in primary culture. Biochim. Biophys. Acta 1500, 153-160.

Meier, P.J., Steger, B., 2002. Bile salt transporters. Annu. Rev. Physiol. 64, 635-661.

Mitsuyoshi, H., Nakashima, T., Sumida, Y., Yoh, T., Nakajima, Y., Ishikawa, H., Inaba, K., Sakamoto, Y., Okanoue, T., Kashima, K., 1999. Ursodeoxycholic acid protects hepatocytes against oxidative injury via induction of antioxidants. Biochem. Biophys. Res. Commun. 263, 537-542.

Miyoshi, H., Rust, C., Roberts, P.J., Burgart, L.J., Gores, G.J., 1999. Hepatocyte apoptosis after bile duct ligation in the mouse involves Fas. Gastroenterology 117, 669-677.

Müller, M., Jansen, P.L.M., 1997. Molecular aspects of hepatobiliary transport. Am. J. Physiol. 272, G1285-G1303.

Nathanson, M.H., Boyer, J.L., 1991. Mechanisms and regulation of bile secretion. Hepatology 14, 551-566.

Neuberger, J., 2000. URSO-panacea or placebo? Hepatology 31, $1027-1028$.

Oh, S.H., Yun, K.J., Nan, J.X., Sohn, D.H., Lee, B.H., 2003. Changes in expression and immunolocalization of protein associated with toxic bile salts-induced apoptosis in rat hepatocytes. Arch. Toxicol. 77, 110-115.

Oliveira, P.J., Esteves, T., Rolo, A.P., Palmeira, C.M., Moreno, A.J., 2004. Carvedilol inhibits the mitochondrial permeability transition by an antioxidant mechanism. Cardiovasc. Toxicol. $4,11-20$.

Ostrow, J.D., 1993. Metabolism of bile salts in cholestasis. In: Tavoloni, N., Berk, P.D. (Eds.), Hepatic Transport and Bile Secretion: Physiology and Pathophysiology. Raven, New York, pp. 673-712.

Oyama, K., Shiota, G., Murawaki, Y., Kawasaki, H., 2002. Reduction of hepatocarcinogenesis by ursodeoxycholic acid in rats. Carcinogenesis 23, 885-892.

Patel, T., Bronk, S.F., Gores, G.J., 1994. Increases of intracellular magnesium promote glycodeoxycholate-induced apoptosis in rat hepatocytes. J. Clin. Invest. 94, 2183-2192.

Patel, T., Gores, G.J., 1997. Inhibition of bile-salt-induced hepatocyte apoptosis by the antioxidant lazaroid U83836E. Toxicol. Appl. Pharmacol. 142, 116-122. 
Plaa, G.L., Priestley, B.G., 1976. Intrahepatic cholestasis by drugs and chemicals. Pharmacol. Rev. 28, 207-273.

Qiao, L., Studer, E., Leach, K., McKinstry, R., Gupta, S., Decker, R., Kukreja, R., Valerie, K., Nagarkatti, P., El Deiry, W., Molkentin, J., Schmidt-Ulrich, R., Fisher, P.B., Grant, S., Hylemon, P.B., Dent, P., 2001. Deoxycholic acid (DCA) causes ligand-independent activation of epidermal growth factor (EGFR) and FAS receptor in primary hepatocytes: inhibition of EGFR/mitogen-activated protein kinase-signaling module enhances DCA-induced apoptosis. Mol. Biol. Cell 12, 2609-2645.

Qiao, L., Yacoub, A., Studer, E., Gupta, S., Pei, X.Y., Grant, S., Hylemon, P.B., Dent, P., 2002. Inhibition of the MAPK and PI3K pathways enhances UDCA-induced apoptosis in primary rodent hepatocytes. Hepatology 35, 779-789.

Rodrigues, C.M.P., Fan, G., Ma, X., Kren, B.T., Steer, C.J., 1998. A novel role for ursodeoxycholic acid in inhibiting apoptosis by modulating mitochondrial membrane perturbation. J. Clin. Invest. 101, 2790-2799.

Rodrigues, C.M., Ma, X., Stieers, C.L., Fan, G., Kren, B.T., Steer, C.J., 1999. Ursodeoxycholic acid prevents cytochrome $c$ release in apoptosis by inhibiting mitochondrial membrane depolarization and channel formation. Cell Death Differ. 6, 842-854.

Rodrigues, C.M., Stieers, C.L., Keene, C.D., Ma, X., Kren, B.T., Low, W.C., Steer, C.J., 2000. Tauroursodeoxycholic acid partially prevents apoptosis induced by 3-nitropropionic acid: evidence for a mitochondrial pathway independent of the permeability transition. J. Neurochem. 75, 2368-2379.

Rolo, A.P., Oliveira, P.J., Moreno, A.J., Palmeira, C.M., 2000. Bile acids affect liver mitochondrial bioenergetics: possible relevance for cholestasis therapy. Toxicol. Sci. 57, 177185.

Rolo, A.P., Oliveira, P.J., Moreno, A.J., Palmeira, C.M., 2001a. Chenodeoxycholate is a potent inducer of the permeability transition pore in rat liver mitochondria. Biosci. Rep. 21, 7380.

Rolo, A.P., Oliveira, P.J., Moreno, A.J., Palmeira, C.M., $2001 \mathrm{~b}$. Protective effect of carvedilol on chenodeoxycholate induction of the permeability transition pore. Biochem. Pharmacol. 61, $1449-1454$.

Rolo, A.P., Oliveira, P.J., Seica, R., Santos, M.S., Moreno, A.J., Palmeira, C.M., 2002a. Improved efficiency of hepatic mitochondrial function in rats with cholestasis induced by an acute dose of $\alpha$-naphthylisothiocyanate. Toxicol. Appl. Pharmacol. 182, 20-26.

Rolo, A.P., Oliveira, P.J., Seica, R., Santos, M.S., Moreno, A.J., Palmeira, C.M., 2002b. Disruption of mitochondrial calcium homeostasis following chronic $\alpha$-naphthylisothiocyanate administration: relevance for cholestasis. J. Invest. Med. 50, 193-200.

Rolo, A.P., Palmeira, C.M., Wallace, K.B., 2002c. Interactions of combined bile acids on hepatocyte viability: cytoprotection or sinergism. Toxicol. Lett. 126, 197-203.

Rolo, A.P., Oliveira, P.J., Moreno, A.J., Palmeira, C.M., 2003a. CDCA induction of mitochondrial permeability transition pore is associated with increased membrane fluidity and cytochrome $c$ release: protective role of carvedilol. Mitochondrion 2, 305311.

Rolo, A.P., Palmeira, C.M., Wallace, K.B., 2003b. Mitochondrially mediated synergistic cell killing by bile acids. Biochim. Biophys. Acta 1637, 127-132.

Rolo, A.P., Palmeira, C.M., Holy, J.M., Wallace, K.B., 2004. Role of mitochondrial dysfunction in combined bile acid-induced cytotoxicity: the switch between apoptosis and necrosis. Toxicol. Sci. 79, 196-204.

Russell, D.W., Setchell, K.D.R., 1992. Bile acid biosynthesis. Biochemistry 31, 4737-4749.

Rust, C., Karnitz, L.M., Paya, C.V., Moscat, J., Simari, R.D., Gores, G.J., 2000. The bile acid taurochenodeoxycholate activates a phosphatidylinositol 3-kinase-dependent survival signaling cascade. J. Biol. Chem. 275, 20210-20216.

Sarbur, C., Kuhajada, K., Keveresan, S., 2001. Evaluation of the lipophilicity of the bile acids and their derivatives by thin-layer chromatography and principal component analysis. J. Chromatogr. A 917, 361-366.

Scholmerich, J., Becher, M.S., Schmidt, K., Schubert, R., Kremer, B., Feldhaus, S., Gerok, W., 1984. Influence of hydroxylation and conjugation of bile salts on their membrane-damaging properties - studies on isolated hepatocytes and lipid membrane vesicles. Hepatology 4, 661-666.

Schroder, O., Rathner, W., Caspary, W.F., Stein, J., 1996. Bile acid-induced increase of rat colonic apical membrane fluidity and proton permeability. Z. Gastroenterol. 34, 365-370.

Shivaram, K.N., Winklhofer-Roob, B.M., Straka, M.S., Devereaux, M.W., Everson, G., Mierau, G.W., Sokol, R.J., 1998. The effect of idebenone, a coenzyme Q analogue, on hydrophobic bile acid toxicity to isolated rat hepatocytes and hepatic mitochondria. Free Rad. Biol. Med. 25, 480-492.

Sodeman, T., Bronk, S.F., Roberts, P.J., Miyoshi, H., Gores, G.J., 2000. Bile salts mediate hepatocyte apoptosis by increasing cell surface trafficking of Fas. Am. J. Physiol. 278, G992-G999.

Sokol, R.J., Devereaux, M., Khandwala, R.A., 1991. Effect of dietary lipid and vitamin $\mathrm{E}$ on mitochondrial lipid peroxidation and hepatic injury in the bile duct-ligated rat. J. Lipid Res. 32, 1349-1357.

Sokol, R.J., Winklhofer-Roob, B.M., Devereaux, M.W., McKim Jr., J.M., 1995. Generation of hydroperoxides in isolated rat hepatocytes and hepatic mitochondria exposed to hydrophobic bile acids. Gastroenterology 109, 1249-1256.

Sokol, R.J., McKim Jr., J.M., Goff, M.C., Ruyle, S.Z., Devereaux, M.W., Han, D., Packer, L., Everson, G., 1998. Vitamin E reduces oxidant injury to mitochondria and the hepatotoxicity of taurochenodeoxycholic acid in the rat. Gastroenterology 114, 164-174.

Solá, S., Brito, M.A., Brites, D., Moura, J.J., Rodrigues, C.M., 2002. Membrane structural changes support the involvement of mitochondria in the bile salt-induced apoptosis of rat hepatocytes. Clin. Sci. 103, 475-485.

Spivey, J.R., Bronk, S.F., Gores, G.J., 1993. Glycochenodeoxycholate-induced lethal hepatocellular injury in rat hepatocytes. J. Clin. Invest. 92, 17-24.

Strupp, W., Weindinger, G., Scheller, C., Ehret, R., Ohnimus, H., Girschick, H., Tas, P., Flory, E., Heinkelein, M., Jassoy, C., 
2000. Treatment of cells with detergents activates caspases and induces apoptotic cell death. J. Membr. Biol. 175, 181-189.

Tanaka, M., Nakura, H., Tateishi, T., Watanabe, M., Nakaya, S., Kumai, T., Kobayashi, S., 1999. Ursodeoxycholic acid prevents hepatic cytochrome $\mathrm{P} 450$ isozyme reduction in rats with deoxycholic acid-induced liver injury. J. Hepatol. 31, 263-270.

Trauner, M., Meier, P.J., Boyer, J.L., 1998. Molecular regulation of hepatocellular transport systems in cholestasis. J. Hepatol. $31,165-178$.

van Hoogstraten, H.J., Hansen, B.E., van Buuren, H.R., ten Kate, F.J., van Berge-Henegouwen, G.P., Schalm, S.W., 1999. Prognostic factors and long-term effects of ursodeoxycholic acid on liver biochemical parameters in patients with primary biliary cirrhosis. Dutch Multi-Centre PBC Study Group. J. Hepatol. 31, 256-262.

Yerushalmi, B., Dahl, R., Devereaux, M.W., Gumpricht, E., Sokol, R.J., 2001. Bile acid-induced rat hepatocyte apoptosis is inhibited by antioxidants and blockers of the mitochondrial permeability transition. Hepatology 33, 616626.

Yue, T.-L., McKenna, P.J., Lysko, P.J., Gu, J.L., Lysco, K.A., Ruffolo, R.R., Feuerstein, G.Z., 1994. SB 211475, a metabolite of carvedilol, a novel antihypertensive agent, is a potent antioxidant. Eur. J. Pharmacol. 251, 237-243.

Zhao, D.L., Hirst, B.H., 1990. Bile salt-induced increases in duodenal brush-border membrane proton permeability, fluidity, and fragility. Dig. Dis. Sci. 35, 589-595. 